Draft version December 27, 2007

Preprint typeset using $\mathrm{LAT}_{\mathrm{E} X} \mathrm{X}$ style emulateapj v. 25/04/01

\title{
NON-EQUILIBRIUM CO CHEMISTRY IN THE SOLAR ATMOSPHERE
}

\author{
A. Asensio Ramos, J. Trujillo Bueno ${ }^{1}$ \\ Instituto de Astrofísica de Canarias, 38200 La Laguna, Tenerife, Spain \\ aasensio@ll.iac.es, jtb@ll.iac.es \\ M. Carlsson \\ Institute for Theoretical Astrophysics, P.O. Box 1029, Blindern, N-0135, Oslo, Norway \\ Mats.Carlsson@astro.uio.no \\ AND \\ J. CERnicharo ${ }^{1}$ \\ Departamento de Astrofísica Molecular e Infrarroja. Instituto de Estructura de la Materia \\ Serrano 123, E-28006 Madrid, Spain \\ cerni@astro.iem.csic.es \\ Draft version December 27, 2007
}

\begin{abstract}
Investigating the reliability of the assumption of instantaneous chemical equilibrium (ICE) for calculating the $\mathrm{CO}$ number density in the solar atmosphere is of crucial importance for the resolution of the long-standing controversy over the existence of 'cool clouds' in the chromosphere, and for determining whether the cool gas owes its existence to $\mathrm{CO}$ radiative cooling or to a hydrodynamical process. Here we report the first results of such an investigation in which we have carried out time-dependent gas-phase chemistry calculations in radiation hydrodynamical simulations of solar chromospheric dynamics. We show that while the ICE approximation turns out to be suitable for modeling the observed infrared $\mathrm{CO}$ lines at the solar disk center, it may substantially overestimate the 'heights of formation' of strong CO lines synthesized close to the edge of the solar disk, especially concerning vigorous dynamic cases resulting from relatively strong photospheric disturbances. This happens because during the cool phases of the hydrodynamical simulations the CO number density in the outer atmospheric regions is smaller than what is stipulated by the ICE approximation, resulting in decreased CO opacity in the solar chromosphere. As a result, the cool CO-bearing gas which produces the observed molecular lines must be located at atmospheric heights not greater than $700 \mathrm{~km}$, approximately. We conclude that taking into account the non-equilibrium chemistry improves the agreement with the available on-disk and off-limb observations, but that the hydrodynamical simulation model has to be even cooler than anticipated by the ICE approximation, and this has to be the case at the 'new' (i.e. deeper) formation regions of the rovibrational CO lines.
\end{abstract}

Subject headings: astrochemistry—molecular processes—radiative transfer-Sun: chromosphere

\section{INTRODUCTION}

Thirty years ago Noyes \& Hall (1972) inferred very low brightness temperatures $(\mathrm{T} \approx 3700 \mathrm{~K})$ from their discovery of strong rovibrational $\mathrm{CO}$ lines at $4.7 \mu \mathrm{m}$ observed close to the edge of the solar disk. Several years later, after such surprising observational results had been confirmed by Ayres \& Testerman (1981), it was suggested that the low chromosphere might not be hot at all but could instead be permeated by CO-cooled 'clouds' at altitudes between 500 and 1000 kilometers above continuum optical depth unity (Ayres 1981). This led to controversy because other (UV and submillimeter) diagnostics had suggested the existence of a uniformly hot chromosphere with a minimum temperature of about $4400 \mathrm{~K}$ near $500 \mathrm{~km}$ and a temperature rise above this temperature-minimum region. The controversy over the existence of cool gas in the low chromosphere continues today (see Kalkofen 2001, Ayres 2002, Avrett 2003), after the publication of an abundance of literature on the subject with theoretical investigations concluding that the CO lines have LTE source functions

${ }^{1}$ Consejo Superior de Investigaciones Científicas (Spain).
(Ayres \& Wiedemann 1989; Uitenbroek 2000a) and with new observations showing off-limb CO emission protruding hundreds of kilometers into the chromosphere (Solanki, Livingston \& Ayres 1994; Clark et al. 1995), but also with the discovery that far-UV chromospheric lines observed on the solar disk always remain in emission at all positions and times (Carlsson, Judge \& Wilhem 1997).

Over the last few years, it has become increasingly evident that the next crucial step towards a better understanding of the enigmatic thermal structure of the solar chromosphere is to carefully investigate the reliability of the assumption of instantaneous chemical equilibrium (ICE), which is currently used for calculating the molecular number densities in stellar atmospheres (see, in particular, Uitenbroek 2000a,b; see also Ayres \& Rabin 1996, Avrett et al. 1996, Asensio Ramos \& Trujillo Bueno 2003, Avrett 2003). Actually, both observations and simulations indicate that the solar chromosphere is a highly inhomogeneous and dynamic region of low density plasma whose thermal, dynamic and magnetic properies we need to decipher for unlocking new discoveries. If the ICE ap- 
proximation turns out to be adequate for modeling the strongest CO lines close to the edge of the solar disk, then the available $\mathrm{CO}$ observations would really be indicating the existence of cool gas in the solar chromosphere. Otherwise, a natural resolution of the current chromospheric temperature discrepancy could perhaps emerge if the $\mathrm{CO}$ concentration turns out to be sufficiently lower than would be expected on the basis of chemical equilibrium. This Letter addresses this challenging issue by showing the first results of a detailed investigation on the non-equilibrium $\mathrm{CO}$ chemistry in the solar atmosphere.

\section{FORMULATION OF THE PROBLEM}

Our strategy consists in performing chemical evolution calculations in the radiation hydrodynamical simulations of solar chromospheric dynamics described by Carlsson \& Stein $(1997,2002)$, that do not include CO cooling in the energy equation. Therefore, at each time step of the hydrodynamical simulation, we have fixed the ensuing thermodynamic conditions and calculated the corresponding CO number density by following the chemical evolution starting from the molecular concentrations of the previous time step.

The chemical evolution (CE) problem consists in calculating the molecular number densities at each time step of the hydrodynamical simulation by taking into account all relevant chemical reactions with their ensuing rates. In order to solve this problem, one has to consider the following evolution equation for each chemical species ' $i$ ' included in the model:

$$
\begin{gathered}
\frac{d n_{i}}{d t}=\sum_{A} \sum_{B} \sum_{C} k_{A B C} n_{A} n_{B} n_{C}+\sum_{A} \sum_{B} k_{A B} n_{A} n_{B}+ \\
\quad+\sum_{A} k_{A} n_{A}-\sum_{A} \sum_{B} k_{A B i} n_{A} n_{B} n_{i}- \\
-\sum_{A} k_{A i} n_{A} n_{i}-k_{i} n_{i}
\end{gathered}
$$

where three-body reactions (first and fourth terms), twobody reactions (second and fifth terms) and one-body reactions (third and sixth terms) have been taken into account. When all the reaction rates for three-body $\left(k_{A B C}\right)$, twobody $\left(k_{A B}\right)$ and one-body reactions $\left(k_{A}\right)$ are given, this set of equations for all the species included in the model form a set of first order ordinary differential equations which has to be solved numerically. Due to the stiffness of the system of equations (produced by the enormous differences in the abundances and their temporal variability), an implicit scheme has to be used. Two key ingredients have to be taken into account: the number of chemical species and the reaction rates for all possible reactions. After a careful investigation, we found that at least the following set of 13 species, which includes the most abundant diatomic molecules, is required to achieve a suitable description of the relevant chemical processes: $\mathrm{H}, \mathrm{C}, \mathrm{O}, \mathrm{N}$, $\mathrm{He}, \mathrm{CH}, \mathrm{CO}, \mathrm{H}_{2}, \mathrm{OH}, \mathrm{NH}, \mathrm{N}_{2}, \mathrm{NO}$ and $\mathrm{CN}$. We have verified that the inclusion of ionic species does not significantly affect the $\mathrm{CO}$ concentration for the typical atmospheric conditions encountered at heights $h \leq 1000 \mathrm{~km}$, although they should be ideally taken into account for very strong shocks capable of producing sizable changes in the degree of ionization. Concerning the reactions rates, we have used a reaction database created for the study of combustion mechanisms (Konnov 2000) which seems to be appropriate for the physical conditions in the solar atmospheric plasma. Other databases like UMIST (Bennet 1988) have been used for the study of the interstellar chemistry, but they suffer from the lack of certain important reactions which can efficiently take place in the Sun's atmosphere (e.g. the catalytic three-body formation of molecular hydrogen: $\mathrm{H}+\mathrm{H}+\mathrm{He} \rightarrow \mathrm{H}_{2}+\mathrm{He}$ ). We have also investigated the possible influence of $\mathrm{CO}$ photodissociations, which are one-body reactions. They are mainly produced by discrete photon absorptions at wavelengths between the Lyman cutoff $(912 \AA)$ and the dissociation threshold (1120 $\mathrm{A})$. By using the photodissociation rates of Mamon, Glassgold \& Huggins (1988), we have verified that the contribution of photodissociation processes to the total $\mathrm{CO}$ concentration is negligible for the radiation field in the solar atmosphere.

\section{RESULTS AND DISCUSSION}

We used two time-series of snapshots from the abovementioned radiation hydrodynamical simulations, each one lasting about 3600 seconds and showing the upward propagation of acoustic wave trains growing in amplitude with height until eventually producing shocks. The first one corresponds to a relatively strong photospheric disturbance showing well-developed cool phases and pronounced hot zones at chromospheric heights (see Carlsson \& Stein 1997). The strongest dynamic cycle of this simulation produces a peak-to-peak line-core brightness temperature of $1000 \mathrm{~K}$, concerning the strong 3-2 R14 CO-line synthesized at disk center with the ICE approximation. However, the brightness temperature variations in most of the cycles of this simulation are $400 \mathrm{~K}$ approximately, which is similar to the observed values found by Uitenbroek, Rabin \& Noyes (1994) under excellent seeing conditions, but larger than those inferred from the temperature histograms in Ayres \& Rabin (1996). We shall refer to this as the strongly dynamic case. ${ }^{2}$ The second simulation corresponds to a much less intense photospheric disturbance (see Carlsson \& Stein 2002), which for strong CO lines synthesized at disk center produces a peak-to-peak linecore brightness temperature fluctuation which is always smaller than $400 \mathrm{~K}$. We shall refer to this as the weakly dynamic case.

Starting from the molecular concentration given by the ICE approximation, we have followed the chemical evolution in order to obtain the temporal variation of the $\mathrm{CO}$ number density $\left(N_{\mathrm{CO}}\right)$ at each height in the simulated solar atmosphere. As expected, the $\mathrm{CO}$ concentration is anticorrelated with the local temperature variations, yielding relatively low $N_{\mathrm{CO}}$ values in the hot phases and relatively high $N_{\mathrm{CO}}$ values in the cool phases. However, the amplitude of the local $N_{\mathrm{CO}}$ fluctuation is smaller than that given by the ICE approximation. In relatively low density regions characteristic of the outer atmospheric layers (e.g. around $h=1000 \mathrm{~km}$ ) the ICE approximation does a fairly good job during the hot phases, but it overestimates the $\mathrm{CO}$ number density during the cool phases. In contrast, in relatively high density regions characteristic of photospheric layers (e.g. around $h=400 \mathrm{~km}$ ) the ICE approximation underestimates the $N_{\mathrm{CO}}$ values during the

2 This is similar to that used by Uitenbroek (2000a) and Ayres (2002) in their ICE modeling, although they considered only a segment of $190 \mathrm{~s}$ corresponding to the most dynamic cycle of the full simulation. 
hot phases. Thus, the $\mathrm{CO}$ abundance does not react instantaneously to the changes in the temperature because of the finite reaction rates. If one carries out a linear analysis by introducing a small temperature perturbation in a medium of given density and temperature and calculates the relaxation time needed to recover the original $\mathrm{CO}$ concentration one finds that, for a given density, the relaxation time is the larger the cooler the medium where the temperature perturbation is introduced. Similarly, for a given temperature of the unperturbed medium, the relaxation time increases rapidly with decreasing density. Short relaxation times are typical of high-temperature and highdensity media (e.g. $t_{\text {relax }} \approx 0.006 \mathrm{~s}$ for $n_{\mathrm{H}}=10^{16} \mathrm{~cm}^{-3}$ and $T=6000 \mathrm{~K}$ ), while long relaxation times are characteristic of low-temperature and low-density situations (e.g. $t_{\text {relax }} \approx 400 \mathrm{~s}$ for $n_{\mathrm{H}}=10^{14} \mathrm{~cm}^{-3}$ and $T=4000 \mathrm{~K}$ ). One would find a broad range of relaxation times at a fixed height (e.g. $h=700 \mathrm{~km}$ ) in the dynamic atmosphere that results from the above-mentioned hydrodynamical simulations, simply because the existing rarefactions, compressions and temperature fluctuations are continually changing the atmospheric conditions. Obviously, the situation is highly non-linear and the relaxation time concept, although useful, loses its meaning in the real Sun. Therefore, any firm conclusion needs to be achieved via detailed numerical simulations.

Fig. 1 refers to the strongly dynamic case. The left panel shows the temporal variation in the brightness temperatures of the line-core emergent intensities at $\mu=1$ and $\mu=0.1$ in the strong 3-2 R14 CO-line. The right panel gives the ensuing fluctuations of the atmospheric height where the line-core optical depth is unity, which we use as an indicator of the 'representative height' where the $\mathrm{CO}$ line-core radiation originates. As seen in the figure, the ICE approximation does a sufficiently good job concerning the synthesis of the emergent $\mathrm{CO}$ spectrum at the solar disk center $(\mu=1)$, but it largely underestimates the line-core emergent intensities at $\mu=0.1$ during the cool phases of the hydrodynamical simulations, producing brightness temperatures that are typically 500 $\mathrm{K}$ lower than those computed with the non-equilibrium $\mathrm{CO}$ concentration. Clearly, this is because during the cool phases the ICE approximation overestimates the 'heights of line formation' by about $300 \mathrm{~km}$, concerning the synthesis of strong $\mathrm{CO}$ lines at $\mu=0.1$ in the strongly dynamic case. This happens because during such cool episodes the $\mathrm{CO}$ number density in the outer atmospheric regions is smaller than what is stipulated by the ICE approximation, resulting in decreased $\mathrm{CO}$ opacity in the solar chromosphere. Interestingly, in the weakly dynamic case which has smaller kinetic temperature fluctuations (but still larger than the fluctuations of observed brightness temperatures!) the ICE approximation does a much better job even at $\mu=0.1$.

The reader may find surprising our conclusion that the ICE approximation is suitable for modeling the $\mathrm{CO}$ spectrum at the solar disk center, given that Uitenbroek (2000b) found that the spatially averaged line cores of weak CO lines synthesized in the three-dimensional (3D) hydrodynamical model of Stein \& Nordlund (1989) are overly dark compared to the observed ATMOS spectrum described by Farmer \& Norton (1989). As we shall show in a forthcoming publication (Asensio Ramos \& Trujillo Bueno; in preparation), this is because such a 3D hydrodynamical model of the solar photosphere is too cold in the CO line forming region. In fact, our ICE synthesis of the 7-6 R68 CO line in the improved 3D hydrodynamical model of Asplund et al. (2000) shows a notable agreement with the observed ATMOS spectrum, which constitutes an additional indication of the realism of the most recent 3D hydrodynamical simulations of solar surface convection (see Shchukina \& Trujillo Bueno 2001 concerning the iron spectrum in such a 3D hydrodynamical model).

Fig. 2 contrasts the time-averaged $\mathrm{CO}$ concentration obtained assuming ICE at each time step of the strongly dynamic simulation case with that resulting from the chemical evolution. Note that the ICE approximation leads to a significant overabundance of $\mathrm{CO}$ in the outer layers of the atmosphere (i.e. above $700 \mathrm{~km}$ ). Thus, the $\mathrm{CO}$ number density can be relatively low in such outer layers, in spite of the fact that the temporally averaged temperature of the Carlsson \& Stein (1997) simulations decreases outwards and does not show any chromospheric temperature rise. As expected from the previously reported results, the ICE approximation does a sufficiently good job concerning the modeling of the temporally-averaged $\mathrm{CO}$ spectrum at the solar disk center $(\mu=1)$. In contrast, the emergent $\mathrm{CO}$ spectrum computed close to the edge of the solar disk (i.e. at $\mu=0.1$ ) shows significantly stronger CO-lines when the ICE approximation is used, especially concerning strong $\mathrm{CO}$ lines like the 3-2 R14 one, for which the line-core brightness temperature is about $100 \mathrm{~K}$ lower than that obtained using the non-equilibrium $\mathrm{CO}$ number densities.

Finally, we turn our attention to the modeling issue of the off-limb $\mathrm{CO}$ emissions, which we have carried out by solving the radiative transfer equation in spherical coordinates at each time step of the hydrodynamical simulation ${ }^{3}$. The off-limb distances where (time-averaged) $\mathrm{CO}$ emission has been observed depend on the line (see Ayres 2002, for a summary of the available off-limb observational results): the off-limb emission extension of strong lines like the 32 R14 line lies between 0.55" and 0.7" above the $4.7 \mu \mathrm{m}$ continuum limb, while weaker lines like the 7-6 R68 line extend to $\sim 0.4$ ". Fig. 3 refers to the strongly dynamic case. It shows that the atmospheric region where we can have a significant off-limb emission is extremely large when the ICE approximation is used, i.e. much larger than when the spectral synthesis is carried out using the non-equilibrium $\mathrm{CO}$ number densities. The dashed and solid lines show the corresponding height variation of the temporally-averaged off-limb emission at the core of the strong 3-2 R14 COline. They show that the non-equilibrium $\mathrm{CO}$ chemistry improves the agreement with the available off-limb observations. First, as seen in Fig. 3, the ICE approximation predicts that appreciable emission in the 3-2 R14 line should continue to relatively large off-limb distances, while partial eclipse measurements by Clark et al (1995) show a

3 This is a suitable strategy for contrasting ICE and non-ICE results, as done also in Fig. 1. It is however clear that a truly realistic modeling of the available observations should take into account the geometrical foreshortening effect resulting from the fact that the solar chromosphere is a highly inhomogeneous medium in which the raypath goes through many different structures. 
rapid disappearance of $\mathrm{CO}$ emission at heights above 700 $\mathrm{km}$, which is more in line with our chemical evolution calculations. Second, the representative off-limb emission extension where the normalized intensity falls to $50 \%$ of the on-disk value is larger when the non-equilibrium $\mathrm{CO}$ concentration is used, which helps to improve the agreement with the observations of translimb emission extensions, although they still seem to be below the most recently observed values (see Ayres 2002).

\section{CONCLUDING COMMENT}

Our results indicate that the $\mathrm{CO}$ line radiation observed close to the edge of the solar disk comes from atmospheric heights not greater than $700 \mathrm{~km}$, approximately, and that the gas in these regions of the low chromosphere must be much cooler than indicated by the cool phases of the Carls- son \& Stein (1997) hydrodynamical simulations. Lower temperatures will probably increase the relaxation times needed to reach the molecular equilibrium concentrations. In a forthcoming publication we will show what happens when the hydrodynamical simulations themselves are carried out taking the $\mathrm{CO}$ cooling into account in a way consistent with the non-equilibrium evolution of the molecular number densities.

\section{ACKNOWLEDGEMENTS}

We thank Gene Avrett, Tom Ayres and Han Uitenbroek for useful remarks. This work has been supported by the Spanish Ministerio de Ciencia y Tecnología through project AYA2001-1649, by the Norwegian Research Council through grant 146467/420 and by the European Commission via the Solar Magnetism Network.

\section{REFERENCES}

Asensio Ramos, A., \& Trujillo Bueno, J. 2003, in Stellar Atmosphere Modeling, ed. I. Hubeny, D. Mihalas \& K. Werner, ASP Conf. Series Vol. 288, in press

Asplund, M., Ludwig, H. G., Nordlund, Å , Stein, R. F. 2000, A\&A, 359,669

Avrett, E. H. 2003, in Current Theoretical Models and Future HighResolution Solar Observations: Preparing for ATST, ed. A. A. Pevtsov \& H. Uitenbroek, ASP Conf. Series, in press

Avrett, E. H., Höflich, Uitenbroek, H. \& Ulmschneider, P. 1996, in Cool Stars, Stellar Systems, and the Sun, 9th Cambridge Workshop, ed. R. Pallavicini and A. Dupree, ASP Conf. Series, Vol. 109, 105

Ayres, T. R. 1981, ApJ, 244, 1064

Ayres, T. R. 2002, ApJ, 575, 1104

Ayres, T. R., \& Testerman, L. 1981, ApJ, 245, 1124

Ayres, T. R., \& Wiedemann, G. R. 1989, ApJ, 338, 1033

Ayres, T. R., \& Rabin, D. 1996, ApJ, 460, 1042

Bennett, A. 1988, in Rate Coefficients in Astrochemistry, ed. T. J. Millar \& D. A. Williams (Dordrecht: Kluwer), 339

Carlsson, M., \& Stein, R. F. 1997, ApJ, 481, 500

Carlsson, M., \& Stein, R. F. 2002, in SOHO-11: From Solar Minimum to Maximum, ed. A. Wilson, ESA SP-508, in press
Carlsson, M., Judge, P. G. \& Wilhem, K. 1997, ApJ, 486, L63

Clark, T. A., Lindsey, C. A., Rabin, D. M., \& Livingston, W. C. 1995, in Infrared Tools for Solar Astrophysics: What's next?, ed. J. Kuhn \& M. Penn (Singapore: World Scientific), 133

Farmer, C. B., \& Norton, R. H. 1989, A High-Resolution Atlas of the Infrared Spectrum of the Sun and the Earth Atmosphere from Space, Vol. 1 (NASA Ref. Pub. 1224)

Kalkofen, W. 2001, ApJ, 557, 376

Konnov, A. A. 2000, Detailed reaction mechanism for small hydrocarbons combustion, http://homepages.vub.ac.be/ akonnov/

Mamon, G. A., Glassgold, A. E., \& Huggins, P. J. 1988, ApJ, 328, 797

Noyes, R. W., \& Hall, D. N. B. 1972, BAAS, 4, 389

Shchukina, N., \& Trujillo Bueno, J. 2001, ApJ, 550, 970

Solanki, S. K., Livingston, W., \& Ayres, T. 1994, Science, 263, 64

Stein, R. F., \& Nordlund, Åi989, ApJ, 342, L95

Uitenbroek, H. 2000a, ApJ, 536, 481

Uitenbroek, H. 2000b, ApJ, 531, 571

Uitenbroek, H., Noyes, R.W. \& Rabin, D. 1994, ApJ, 432, L67 

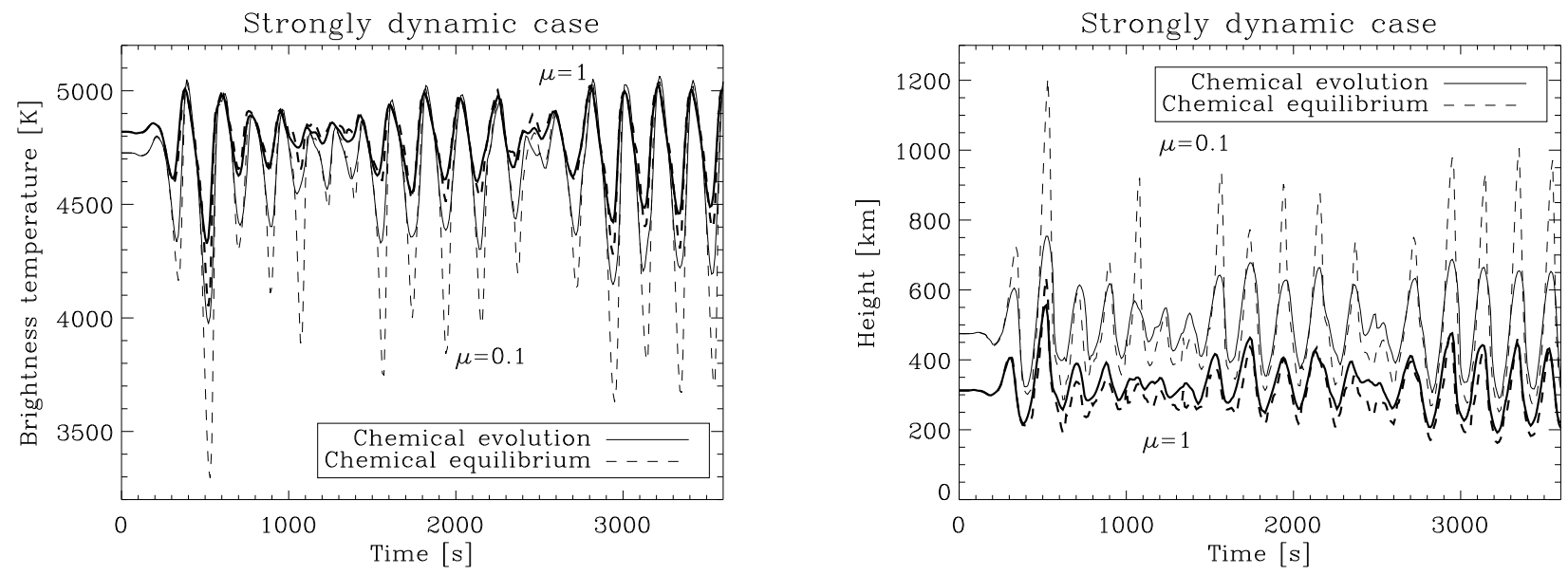

FIG. 1.- Temporal variation of the brightness temperature in the core of the strong 3-2 R14 line (left panel) and of the height of line-core optical depth unity (right panel) for the strongly dynamic case and for two observing angles: disk center ( $\mu=1$, heavy lines) and close to the solar limb ( $\mu=0.1$, light lines), where $\mu=\cos \theta$ (with $\theta$ the angle between the solar radius vector and the line of sight).

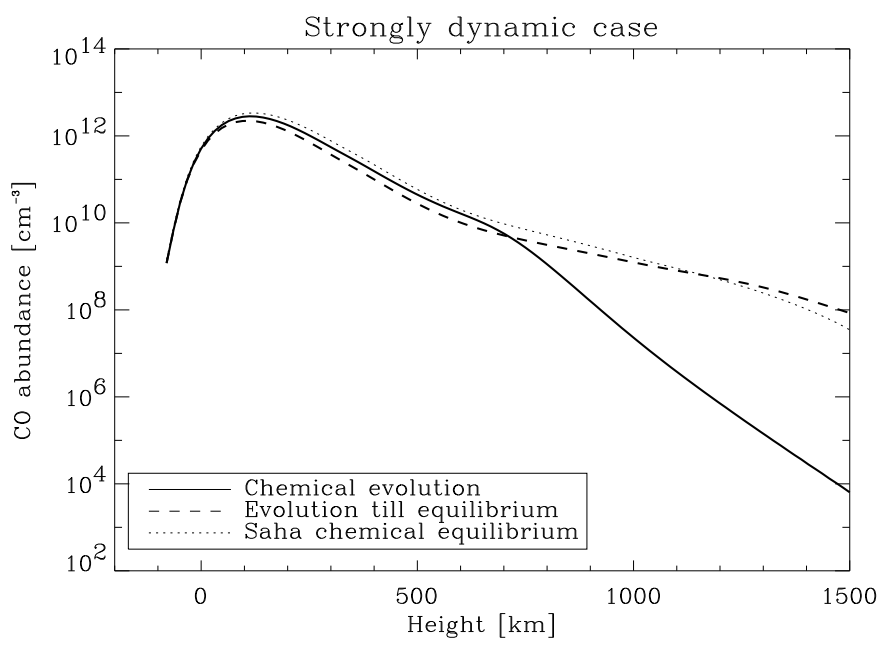

FIG. 2.- Solid line: the height variation of the time-averaged CO concentration obtained from the chemical evolution calculation in the strongly dynamic simulation case. Dashed line: time-averaged CO concentration corresponding to the ICE approximation, but calculating the CO number densities of the atmospheric models associated to each time step by using the same chemical evolution code until reaching the ensuing equilibrium concentrations. Dotted line: time-averaged CO concentration corresponding to the ICE approximation, but calculating the $\mathrm{CO}$ concentrations directly from the Saha chemical equilibrium equations. A comparison of the dashed and dotted lines illustrates the reliability of the chosen database for the chemical evolution calculations. In any case, in order to be fully consistent with our comparisons, all ICE results in this paper refer to 'evolution until equilibrium' calculations. 


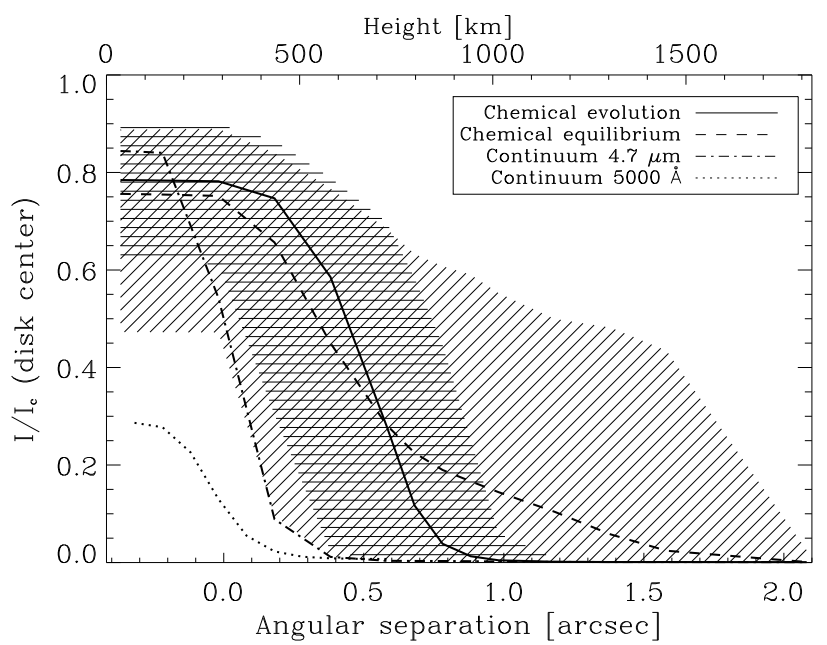

FIG. 3.- The diagonal shading indicates the relatively large atmospheric region where the ICE approximation predicts off-limb emission in the core of the 3-2 R14 CO-line, while the horizontal shading shows that corresponding to the chemical evolution calculation. The figure also shows the calculated limb profiles for the continua at $4.7 \mu \mathrm{m}$ and $5000 \AA$. The zero level of the angular separation scale refers to the 4.7 $\mu \mathrm{m}$ continuum edge calculated as the $50 \%$ point in the intensity drop, as normalized to the ensuing continuum intensity at disk center. At each angular distance, the vertical extensions of the shadings indicate the amplitude of the temporal variation of the line-core CO-emission. The dashed and solid lines give the corresponding temporally averaged off-limb emissions. The upper horizontal axis gives the height in the atmosphere, $z=0 \mathrm{~km}$ being the location of continuum optical depth unity at $5000 \AA$ for a disk center observation (i.e., the usual depth scale in solar models). Note that at low altitude locations, where the IR continuum dashed-dotted curve lies above the (temporally-averaged) dashed and solid curves, the synthesized CO line turns into an absorption line for the ICE and non-equilibrium cases, respectively. 